January 2013

\title{
Self-management education interventions for persons with schizophrenia: a meta-analysis
}

Haiou Zou

Peking Union Medical College, School of Nursing

Zheng Li

Peking Union Medical College, School of Nursing

Marie T Nolan

Johns Hopkins University, Baltimore, Maryland, USA

David Arthur

Aga Khan University, david.arthur@aku.edu

Hongxing Wang

Capital Medical University, Beijing, China

See next page for additional authors

Follow this and additional works at: https://ecommons.aku.edu/pakistan_fhs_son

Part of the Nursing Midwifery Commons

\section{Recommended Citation}

Zou, H., Li, Z., Nolan, M., Arthur, D., Wang, H., Hu, L. (2013). Self-management education interventions for persons with schizophrenia: a meta-analysis. International Journal of Mental Health Nursing, 22(3), 256-271.

Available at: https://ecommons.aku.edu/pakistan_fhs_son/211 
Authors

Haiou Zou, Zheng Li, Marie T Nolan, David Arthur, Hongxing Wang, and Lili Hu 


\title{
FEATURE ARTICLE
}

\section{Self-management education interventions for persons with schizophrenia: A meta-analysis}

\author{
Haiou Zou, ${ }^{1}$ Zheng Li, ${ }^{1}$ Marie T. Nolan, ${ }^{3}$ David Arthur, ${ }^{4}$ Hongxing Wang ${ }^{2}$ and Lili Hu ${ }^{2}$ \\ ${ }^{1}$ Peking Union Medical College, School of Nursing, ${ }^{2}$ Beijing An Ding Hospital, Capital Medical University, Beijing, \\ China; ${ }^{3}$ School of Nursing, Johns Hopkins University, Baltimore, Maryland, USA; and ${ }^{4}$ Angeles University \\ Foundation, Angeles City, the Philippines
}

\begin{abstract}
Although self-management education programs for persons with schizophrenia are being developed and advocated, uncertainty about their overall effectiveness remains. The purpose of this meta-analysis was to examine outcomes of self-management education interventions in persons with schizophrenia. Six electronic databases were searched. Manual searches were conducted of the reference lists of the identified studies and major psychiatric journals. Randomized controlled trials of self-management education interventions aimed at reducing relapse and hospital readmissions, as well as improving symptoms, psychosocial functioning, and adherence to medication treatment were identified. Data were extracted and the quality of included studies were rated by two authors independently. Finally, 13 studies with 1404 patients were included. Self-management education interventions were associated with a significant reduction of relapse events and re-hospitalizations. Patients who received self-management education were more likely to improve adherence to medication and symptoms compared to patients receiving other care. However, a benefit on psychosocial functioning was not confirmed in the current meta-analysis. The study concludes that self-management education intervention is a feasible and effective method for persons with schizophrenia and should be routinely offered to all persons with schizophrenia.
\end{abstract}

KEY WORDS: meta-analysis, schizophrenia, self-management education interventions.

\section{INTRODUCTION}

Schizophrenia is a chronic, severe, and lifelong brain disorder that affects approximately $0.7 \%$ of the population with an incidence rate of 3 per 10000 persons (WHO 2001). Despite access to the benefits of second-generation psychopharmacotherapy, persons with

Correspondence: Zheng Li, Peking Union Medical College, School of Nursing, Dong Dan San Tiao, No.9, Dong Cheng District, Beijing 100730, China. Email: zhengli@hotmail.com

Haiou Zou, Mphil.

Zheng Li, PhD, Professor and Associate Dean

Hongxing Wang, MD, Professor.

Lili Hu, BSN, Director of Nursing.

Marie T. Nolan, PhD, Professor.

David Arthur, PhD, Professor.

Conflict of interest: The authors report no actual or potential conflicts of interest.

Accepted June 2012 schizophrenia still experience persistent psychotic symptoms (Kane 1996; Lindenmayer 2000), impaired social functioning (Angell \& Test 2002; Norman et al. 1999), unsatisfactory quality of life (Kopelowicz et al. 2003), and low employment (McGurk \& Mueser 2004; Racenstein et al. 2002). Thus, it is not surprising that schizophrenia is listed as the eighth leading cause of disability-adjusted life years (DALY) worldwide in the age group 15-44 years, and is expensive to manage. Its estimated economic cost in the USA, for example, was $\$$ US 62.7 billion in 2002 of which \$US 22.7 billion was excess direct care cost $(\mathrm{Wu}$ et al. 2005).

Psychosocial interventions are offered to persons with schizophrenia in order to improve on the benefits of psychopharmacotherapy. One of them is self-management skills training (Mueser \& McGurk 2004). The intervention is designed to improve knowledge, management of 
symptoms, social and occupational functioning, and to lower risk of relapse (Newman et al. 2004).

As early as 2002, it was accepted that persons with schizophrenia could participate in managing their illness, and thus, could possibly benefit from psychoeducation. Although self-management education programs for persons with schizophrenia are becoming increasingly common, there is still uncertainty about their overall effectiveness. Recent research has examined the benefits of self-management education in this population. However, there has been no known systematic review of existing evidence of the efficacy or effectiveness of educational interventions to advance the self-management skills of persons with schizophrenia. The purpose of this meta-analysis was to examine outcomes of selfmanagement education intervention in persons with schizophrenia. Outcomes assessed included relapse or re-hospitalization, medication adherence, psychiatric symptoms, and psychosocial functioning.

\section{BACKGROUND AND CLINICAL FRAMEWORK}

The terms 'psychoeducation' and 'self-management education' are closely related concepts. Bodenheimer et al. (2002) distinguished between psychoeducation, which they suggested provides disease-specific information, and self-management education which teaches problemsolving skills which allow patients to take appropriate actions to improve their health (Bodenheimer et al. 2002). Psychoeducational interventions have been found to help persons gain basic knowledge of their illness (Vreeland et al. 2006). However, psychoeducation alone does not appear to help clients manage their illness better or engage in the recovery process as a consequence of this newly gained knowledge (Mueser \& McGurk 2004; Vreeland et al. 2006). Self-management education provides both education and practical selfmanagement skills to promote active illness management (e.g. altering medication, monitoring symptoms, or seeking help).

The aim of self-management education is to facilitate the ability of the learner to carry out disease-specific medical regimens, guide health behaviour change, and provide emotional support for patients so that they can better manage their disease and live functional lives (Bourbeau et al. 2003). According to Mueser and McGurk (2004), effective self-management education in schizophrenia includes four areas of content: medication management, recognition of early warning signs of relapse, development of a relapse prevention plan, and coping skills for dealing with persistent symptoms (Mueser \& McGurk 2004).

In the case of schizophrenia, it is argued that if patients learn basic facts about schizophrenia and its management, they will be able to make informed decisions about their care (Atkinson et al. 1996). Second, if they know how to recognize and appropriately manage early warning signs of relapse and develop a plan to respond, patients can learn to prevent relapse. Third, once patients are taught coping skills, they can use these to deal with persistent symptoms, the effects of which should become evident in terms of relapse and re-hospitalization.

\section{METHODS}

\section{Study selection}

This meta-analysis addressed those teaching intervention studies that implemented self-management education in all four areas of content: (i) medication management; (ii) recognition of early warning signs of relapse; (iii) development of a relapse prevention plan; and (iv) coping skills for dealing with persistent symptoms. The meta-analysis did not select studies that addressed other psychological intervention foci such as cognitive behaviour therapy, assertive community treatment, social skills training, or family intervention. Studies meeting the following criteria were selected for this meta-analysis:

1 Randomized controlled trials (RCT).

2 Adult participants aged 18 years or more.

3 Participants with a diagnosis of schizophrenia according to the International Classification of Diseases, 10th Revision (ICD-10).

4 Control group participants that received either standard care or were on a wait list.

5 Outcome measures of relapse, re-hospitalization, adherence to psychotropic medication regimen, or psychiatric symptoms, or psychosocial functioning.

6 Studies had been reviewed by an institutional ethics committee prior to implementation.

Studies were excluded if they did not include all four areas of teaching content recommended by Mueser and McGurk (2004). Studies were also excluded if the intervention provided only basic information about schizophrenia and its treatment, or focused solely on improving adherence to psychopharmacotherapy (e.g. Meder et al. 1998), or did not provide all information needed for estimating effect size (e.g. Anzai et al. 2002), that is, the number of subjects in each comparison group and the mean and standard deviation of each outcome assessed. 
Studies which included peer-led self-management were also excluded.

\section{Data sources and search protocol}

Electronic databases including the Cochrane Central Register of Controlled Trials (CENTRAL), PubMed, CINAHL, EMBASE, PsycINFO, and Web of Science were searched from 1996 to December 2010. The current study only included papers written in English. The search term 'schizophrenia' was used to identify the disorder. Because self-management can be labelled with different terms, the following terms were used: 'self-manag*' or 'self manag*' or 'disease manag*' or 'self-car*' or 'train*' or 'instruct*' or 'teach*' or 'educat*' or 'management plan*' or 'management program*' or 'empowerment' or 'health promotion' or 'complian*' or 'adheren*' or 'elapse' or 'symptom manag*' or 'medication manag*'. The reference lists of the identified studies were inspected to identify additional eligible publications. Major journals (e.g. Archives of General Psychiatry, The American Journal of Psychiatry, Schizophrenia Research, Schizophrenia Bulletin, British Journal of Psychiatry, Current Opinion in Psychiatry, and Patient Education and Counseling) also were hand-searched for the same reason.

\section{Data extraction and quality assessment}

Two authors (H. Z. and Z. L.) independently reviewed all studies to determine if they met the inclusion and exclusion criteria and to rate the quality of the included studies. If there was disagreement, consensus was reached through discussion. If consensus was not reached a third author was consulted (H. W.) and the decision of two of the three authors was adopted. Two authors (H. Z. and Z. L.) independently extracted the data from the studies selected for meta-analysis. Information included the location of study, recruitment strategies, study design, participants' characteristics, intervention (e.g. type, content, intensity, duration, and follow up), and outcomes. If there was disagreement during data extraction, a third author (H. W.) was approached and consensus was reached through discussion. The reviewers also contacted three authors of studies for missing outcome data.

The Jadad scale was used to assess the methodological quality of the included studies because there is no consensus on a gold standard to evaluate the internal validity or methodological quality of an RCT. Accordingly, the selection of the Jadad scale has relative merit because it assesses the most important individual components of methodological quality by using a simple approach. This nominal scale addresses three essential questions and two other questions:
1 Was the study described as randomized?

2 Was the study described as double-blind?

3 Was there a description of withdrawals and dropouts?

4 Was there a description of the method of randomization, and was the method appropriate for the study?

5 Was there a description of the method for blinding, and was the method appropriate?

A point is assigned to a study if there is a positive answer to a question. Thus, a Jadad score for the methodological quality of a study can range from 0 (i.e. very poor) to 5 (i.e. rigorous).

\section{Statistical analysis}

The Review Manager software (RevMan, 2003), provided by The Cochrane Collaboration (Oxford, UK) was used to conduct the meta-analysis.

\section{Heterogeneity test}

Heterogeneity between studies was explored by using the $\mathrm{I}^{2}$-test of heterogeneity which indicates the percentage of inconsistency due to heterogeneity. The higher the percentage, the higher the level of heterogeneity or variability of the outcome variable measures (Higgins et al. 2003).

\section{Effect size analysis}

The effect size in meta-analysis reflects the magnitude of the treatment effect or the strength of a relationship between two variables and is the unit in a meta-analysis. In this study, the effect size for each study and summary effect were both computed. Effect sizes based on dichotomous data (e.g. relapse/re-hospitalization, adherence to psychotropic medication regimen) in this meta-analysis were calculated by using odds ratios (OR). OR are the odds of success in the intervention group relative to the odds of success in the control group. Effect sizes based on continuous data (e.g. psychiatric symptoms) were calculated by using Weighted Mean Difference (WMD) with 95\% confidence intervals $(\mathrm{CI})$.

To compute the effect size, fixed-effect model and 95\% CI were adopted. Under the fixed-effect model, it is assumed that all studies come from a common population, and that the effect size is not significantly different among the different trials. This assumption is tested by the heterogeneity test'. An I $\mathrm{I}^{2}$ less than $50 \%$ was taken to indicate mild to moderate heterogeneity, and a fixed-effect model was used to synthesis the results. An I ${ }^{2}$ of more than $50 \%$ was taken as notable heterogeneity and a random-effects model was used to summarize the results (Higgins et al. 2003), in which both the random variation within the 
studies and the variation between the different studies are incorporated. If using the random-effects model did not make a difference and inconsistency remained high, data were presented separately and reasons for heterogeneity investigated.

\section{RESULTS}

\section{Results of the search}

The search yielded titles of 2276 papers. Abstracts of the studies were reviewed if their title pointed to some assessment of a self-management educational intervention. This process yielded 30 relevant papers for inspection of which 17 were excluded for the following reasons:

1 The studies did not provide sufficient information $(n=3)$.

2 The intervention examined did not include the four criterion areas of teaching content required for the meta-analysis $(n=3)$.

3 The outcomes assessed were not relevant outcome measures of the intervention $(n=7)$, or the relevant outcomes assessed did not include relapse or rehospitalization, adherence to psychotropic medication, psychiatric symptoms, and psychosocial functioning.

4 The studies did not report complete information needed for estimating effect size data $(n=4)$.

Altogether, 13 RCT met the criteria for inclusion in the meta-analysis (Fig 1). All studies were reviewed by an institutional ethics committee prior to implementation. Information about the studies is shown in Table 1, organized by author, country and year of publication, method, intervention examined, participant characteristics, outcomes assessed and their measures, study findings, and Jadad score. Information about each RCT included the number of participants in the intervention or control group, and time of follow-up assessment. Information about intervention included the mode, content, and duration of the intervention. Participant characteristics included location of recruitment, sex, and number of participants who completed or dropped out of a study. Findings identified included relapse or re-hospitalization, medication adherence, psychiatric symptoms, and psychosocial functioning.

\section{Study characteristics}

The 13 RCT were conducted in the China $(n=4)$, the USA $(n=3)$, Germany $(n=2)$, France $(n=1)$, UK $(n=1)$, Denmark $(n=1)$, and Mexico $(n=1)$. Participants were recruited from an inpatient setting $(n=3)$, outpatient setting $(n=7)$, or community mental health

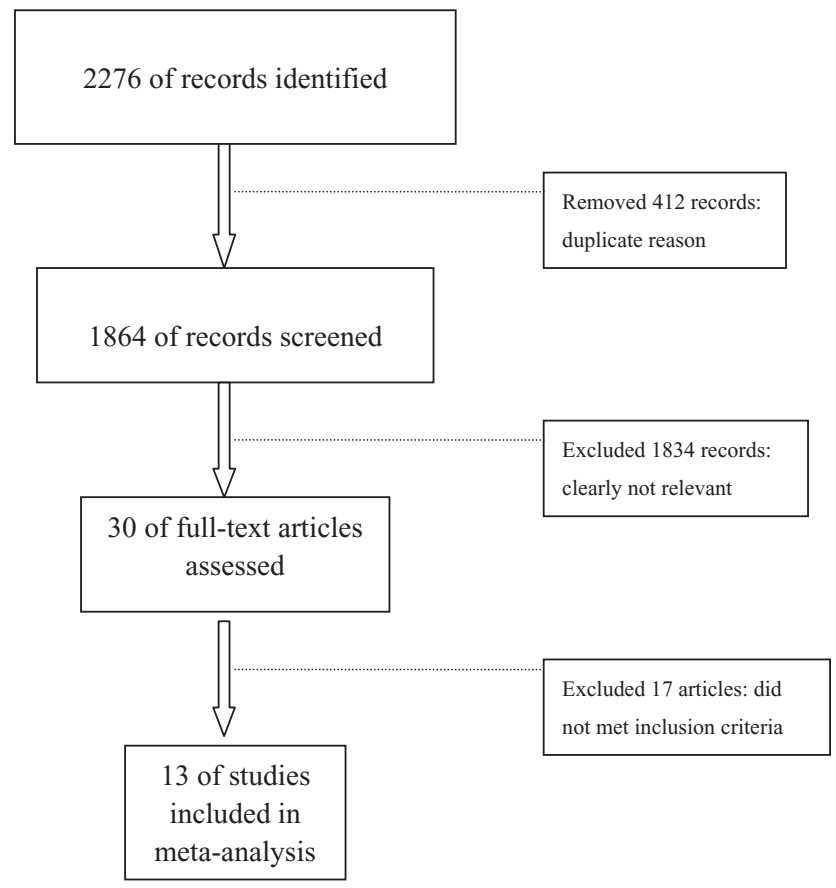

FIG. 1: Flowchart of search results.

centres $(n=3)$. The number of participants in the 13 studies ranged 46-236 for a total of 1404 participants (Table 1). In the self-management education intervention group, the number of participants ranged 23-125. Similarly, in the control group, the number of participants ranged 23-111. Males comprised $42-100 \%$ of the samples. (In the intervention group, males comprised $29.2-100 \%$. In the control group, males comprised $38-100 \%$.) The mean age of the participants in the intervention group ranged 29.7-39.5 years. Similarly, in the control group, the mean age of the participants ranged 30.1-40 years. Attrition rates in the two groups were comparable. In the intervention group, attrition rates varied from $0-37 \%$, while in the control group, attrition rates ranged $0-33 \%$.

In the trials, a total of 726 participants were assigned to self-management education intervention and 678 participants to the control group. All interventions were delivered in a group setting. The number of intervention sessions delivered ranged 7 (Chabannes et al. 2008) to 48 (Kopelowicz et al. 2003; Valencia et al. 2007), each one lasting from $45 \mathrm{~min}$ (Xiong et al. 1994) to 1.5 hours (Kopelowicz et al. 2003). The time of follow-up assessments ranged from immediately post-intervention (Shin \& Lukens 2002; Vreeland et al. 2006) to 24 months postintervention (Chabannes et al. 2008; Pitschel-Walz et al. 2006). 


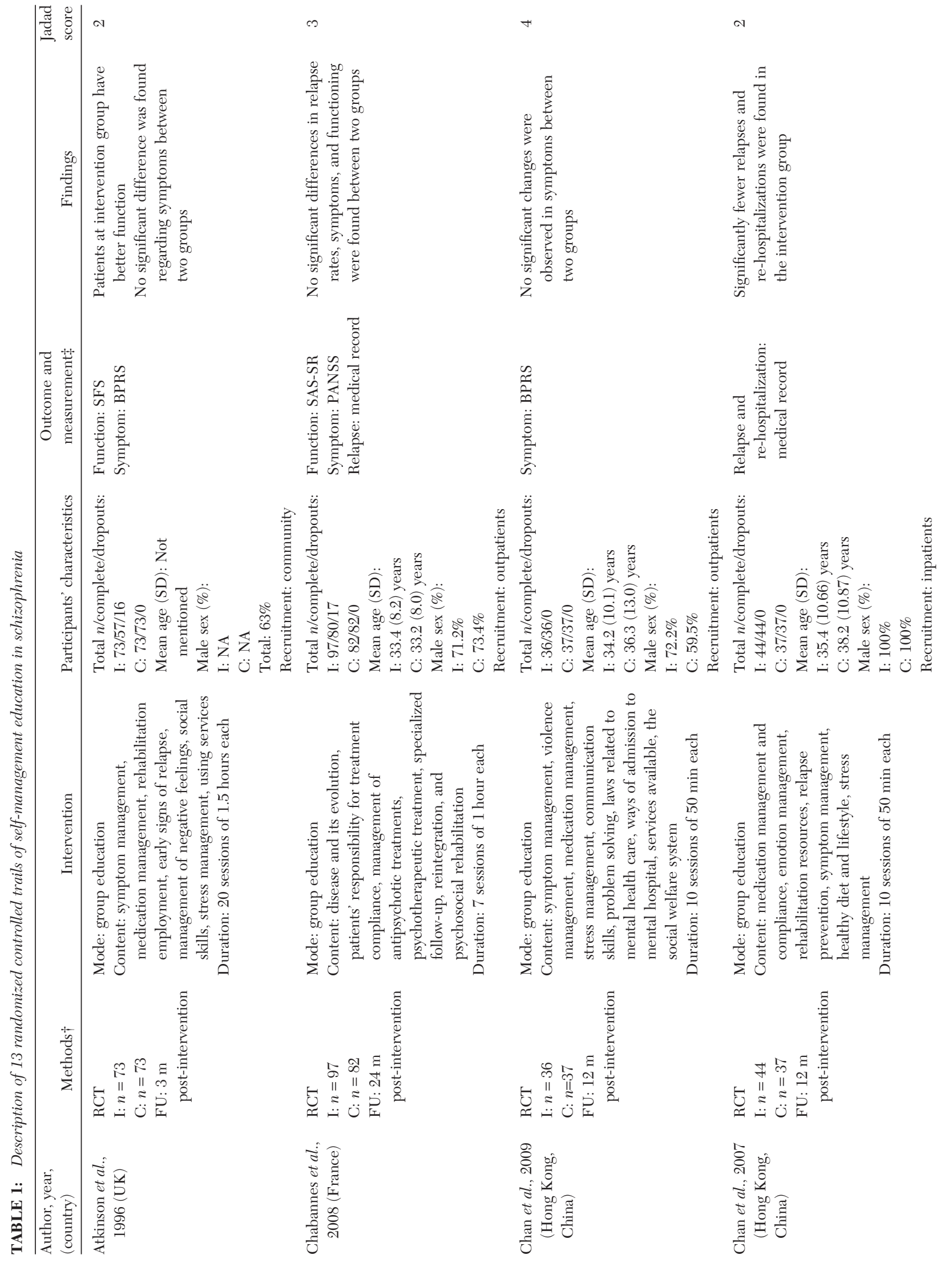

(C) 2012 The Authors 

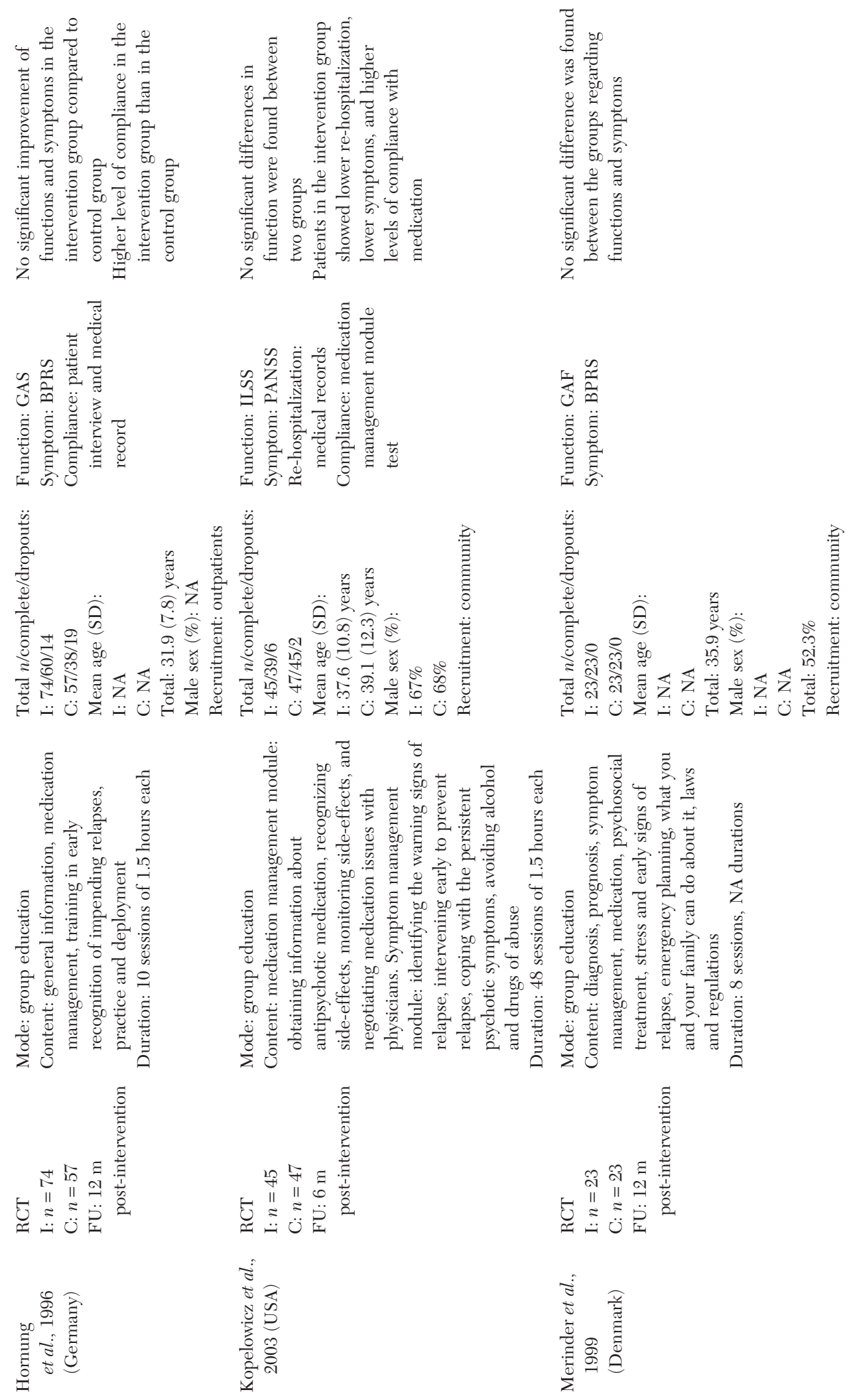


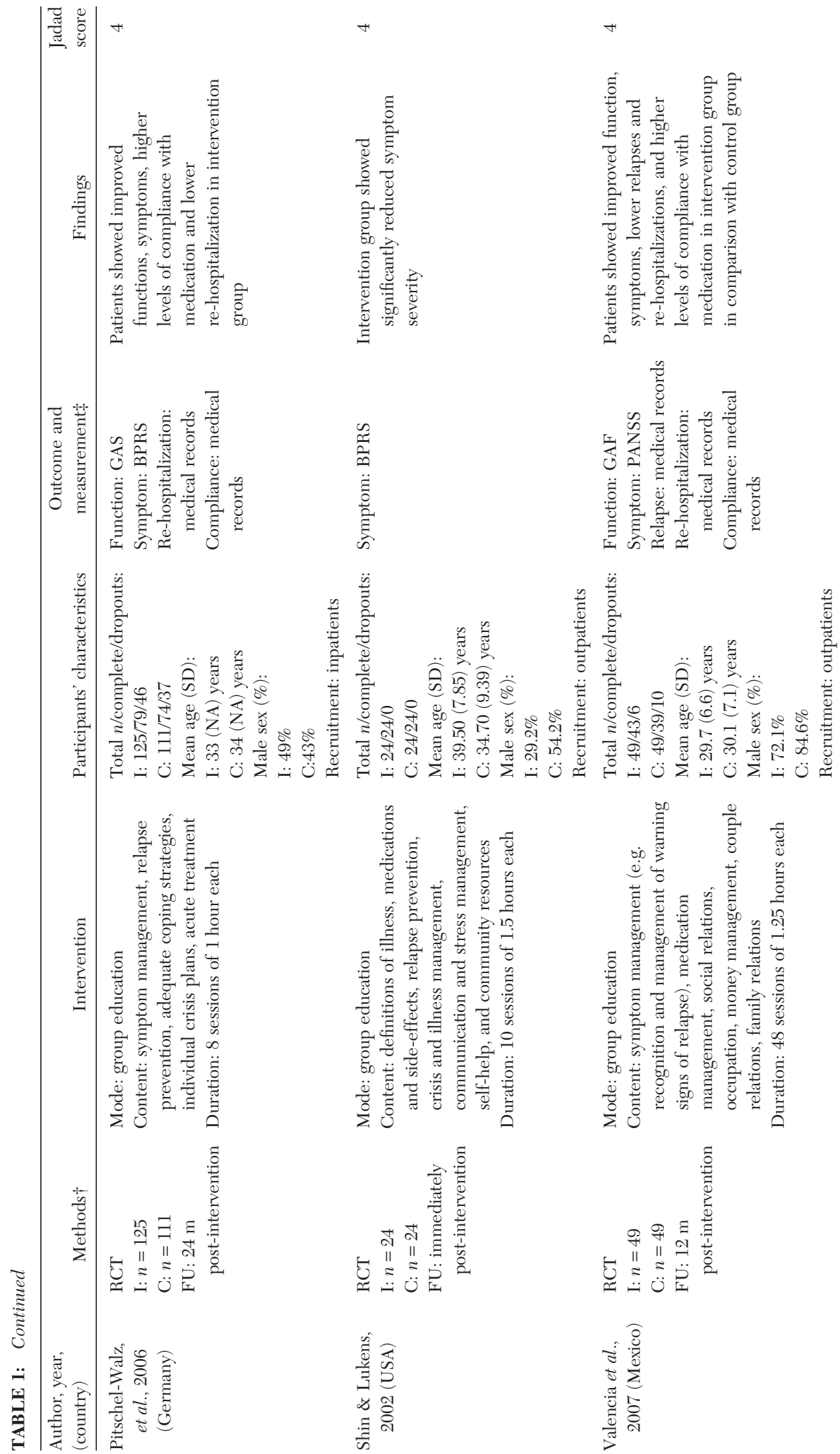

(C) 2012 The Authors International Journal of Mental Health Nursing (c) 2012 Australian College of Mental Health Nurses Inc. 

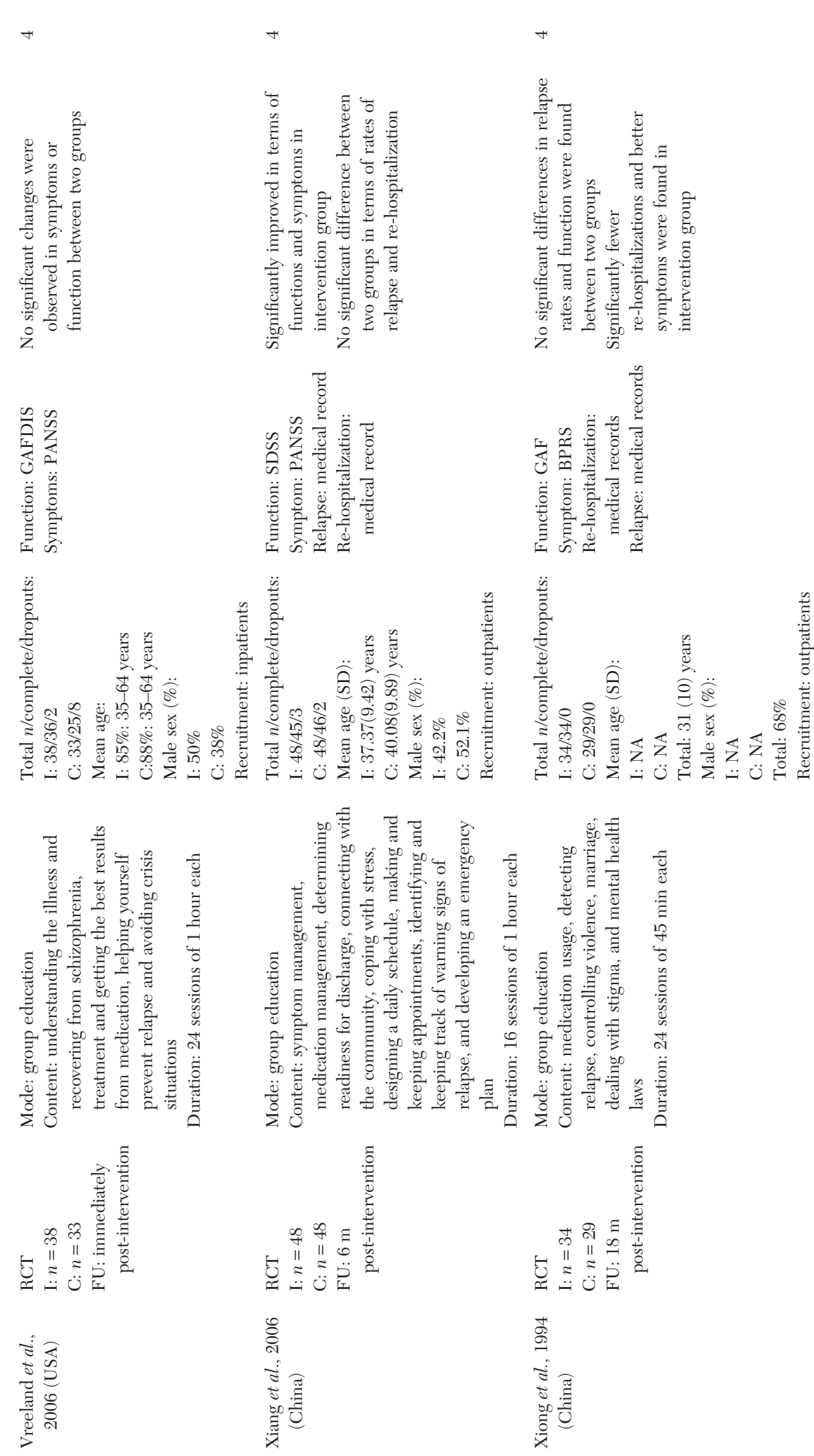
TABLE 2: Total effect sizes and confidence intervals for relapse, re-hospitalization, adherence to medication, and psychiatric symptoms (comparison between self-management education intervention and treatment as usual)

\begin{tabular}{|c|c|c|c|c|c|}
\hline & & $n$ & & & \\
\hline Outcome category & $k$ & $\begin{array}{l}\text { Self-management } \\
\text { education } \\
\text { intervention }\end{array}$ & $\begin{array}{c}\text { Treatment } \\
\text { as usual }\end{array}$ & Total effect size $(95 \% \mathrm{CI}) \dagger$ & $P$ \\
\hline Relapse & 5 & 275 & 259 & $\mathrm{OR}=0.54(0.36-0.83)$ & 0.004 \\
\hline Re-hospitalization & 7 & 392 & 379 & $\mathrm{OR}=0.55(0.39-0.77)$ & 0.000 \\
\hline Adherence to medication & 4 & 229 & 206 & $\mathrm{OR}=2.57(1.57-4.19)$ & 0.000 \\
\hline Psychiatric symptoms: measured by PANSS & 3 & 127 & 130 & $\begin{array}{l}\text { WMD: } \\
\text { PANSS positive: }-2.65(-3.62 \text { to }-1.67) \\
\text { PANSS negative: }-4.01(-5.23 \text { to }-2.79) \\
\text { PANSS general: }-3.39(-4.50 \text { to }-2.29)\end{array}$ & 0.000 \\
\hline Psychiatric symptoms: measured by BPRS & 5 & 222 & 187 & WMD: $-4.19(-5.84$ to -2.54$)$ & 0.000 \\
\hline
\end{tabular}

$\dagger$ Integration carried out in fixed effects model. BPRS, Brief Psychiatric Rating Scale; CI, confidence interval; $k$, number of included studies; $n$, number of investigated participants; OR: odds ratio; $P$, level of significance for total effect size; PANSS, Positive and Negative Syndrome Scale; WMD, Weighted Mean Difference.

\section{Risk of bias in included studies}

Five studies contained a description of the method of randomization (Chabannes et al. 2008; Hornung et al. 1996; Merinder et al. 1999; Pitschel-Walz et al. 2006; Vreeland et al. 2006). Three studies did not provide information about blinding (Atkinson et al. 1996; Chabannes et al. 2008; Chan et al. 2007). One of the 10 blinded studies did not provide information about its method of blinding (Hornung et al. 1996). All studies provided attrition data and reasons for participant withdrawal from a study. However, no study was double-blinded. Therefore, no study scored 5 according to the Jadad score. Nine of the 13 studies scored 4 points (Chan et al. 2009; Kopelowicz et al. 2003; Merinder et al. 1999; Pitschel-Walz et al. 2006; Shin \& Lukens 2002; Valencia et al. 2007; Vreeland et al. 2006; Xiang et al. 2006; Xiong et al. 1994). Two scored 3 points (Chabannes et al. 2008; Hornung et al. 1996), and two studies scored 2 points (Atkinson et al. 1996; Chan et al. 2007).

\section{Efficacy of self-management education intervention}

\section{Relapse}

Five studies reported outcome data on relapse (Chabannes et al. 2008; Chan et al. 2007; Valencia et al. 2007; Xiang et al. 2006; Xiong et al. 1994). The results revealed that there was no heterogeneity among the five studies $\left(\mathrm{I}^{2}=0 \%\right)$. Overall, 53 participants relapsed in the self-management education intervention group (total, $n=275)$ and 76 participants relapsed in the treatment as usual group (total, $n=259$ ). OR were used to compare the relative odds of relapse in the two groups, and those receiving a self-management education intervention were found to be $46 \%$ less likely to experience relapse than those receiving treatment as usual $(\mathrm{OR}=0.54,95 \%$ $\mathrm{CI}=0.36-0.83, P=0.004)$ (Table 2). Data on effect size for individual studies is available from the authors.

\section{Re-hospitalization}

Seven studies (Chabannes et al. 2008; Chan et al. 2007; Kopelowicz et al. 2003; Pitschel-Walz et al. 2006; Valencia et al. 2007; Xiang et al. 2006; Xiong et al. 1994), with a combined total of 771 participants, reported outcomes on re-hospitalization with no heterogeneity between the studies $\left(\mathrm{I}^{2}=0 \%\right)$. According to the results, there were 92 participants re-hospitalized in the self-management education intervention group (total, $n=392$ ) and 130 participants re-hospitalized in the treatment as usual group (total, $n=379$ ). The odds of fewer re-hospitalizations favoured patients receiving self-management education intervention. Participants receiving self-management education intervention were $45 \%$ less likely to experience re-hospitalization than those receiving treatment as usual $(\mathrm{OR}=0.55,95 \% \mathrm{CI}=0.39-0.77, P=0.000)($ Table 2$)$.

\section{Adherence to medication}

Four studies (Hornung et al. 1996; Kopelowicz et al. 2003; Pitschel-Walz et al. 2006; Valencia et al. 2007), with a combined total of 435 participants, reported outcomes on adherence to medication. Meta-analysis of the data revealed greater adherence in participants receiving the self-management education intervention compared to those receiving treatment as usual $(\mathrm{OR}=2.57,95 \%$ $\mathrm{CI}=1.57-4.19, P=0.000)($ Table 2$)$. There was a 2.57 fold greater odds of adherence to medication for participants receiving self-management intervention than for those receiving treatment as usual. 


\section{Psychiatric symptoms}

The effect of the self-management education intervention on participants' psychiatric symptoms was assessed in 12 studies (Atkinson et al. 1996; Chabannes et al. 2008; Chan et al. 2009; Hornung et al. 1996; Kopelowicz et al. 2003; Merinder et al. 1999; Shin \& Lukens 2002; Valencia et al. 2007; Vreeland et al. 2006; Xiang et al. 2006; Xiong et al. 1994). Symptom data from three studies were not included in the meta-analysis because the authors did not provide information about means or standard deviations (Atkinson et al. 1996; Chabannes et al. 2008; Xiong et al. 1994).

Five studies reported mean data for psychiatric symptoms using the Brief Psychiatric Rating Scale (BPRS) (Chan et al. 2009; Hornung et al. 1996; Merinder et al. 1999; Pitschel-Walz et al. 2006; Shin \& Lukens 2002). Meta-analysis of these data demonstrated that participants receiving the self-management education intervention were significantly more likely to demonstrate a reduction in severity of symptoms (WMD $=-4.19,95 \%$ $\mathrm{CI}=-5.84$ to $-2.54, P=0.000)$ (Table 2).

Four studies (Kopelowicz et al. 2003; Valencia et al. 2007; Vreeland et al. 2006; Xiang et al. 2006) reported mean data for psychiatric symptoms measured with the Positive and Negative Syndrome Scale (PANSS), involving 318 participants; however, there was substantial heterogeneity (positive subscale $\mathrm{I}^{2}=78.7 \%$; negative subscale $\mathrm{I}^{2}=86.4 \%$; general subscale $\mathrm{I}^{2}=62.3 \%$ ) between the studies. A random-effects model was therefore adopted, in which both the random variation within the studies and the variation between the different studies was incorporated. However, after using the random-effects model, results of the analysis continued to demonstrate high levels of heterogeneity. Therefore, the authors examined individual studies and removed one that appeared problematic (Vreeland et al. 2006) from the meta-analysis. When the Vreeland et al. (2006) study was removed, heterogeneity reduced significantly (positive subscale $\mathrm{I}^{2}=40.3 \%$; negative subscale $\mathrm{I}^{2}=29.7 \%$; general subscale $\mathrm{I}^{2}=61.9 \%$ ). A meta-analysis was carried out with the three remaining studies (Kopelowicz et al. 2003; Valencia et al. 2007; Xiang et al. 2006) involving 257 participants. Again, the results showed a significant reduction in severity of psychiatric symptoms in favour of the self-management education intervention group in positive symptoms (WMD $=-2.12$, $95 \% \mathrm{CI}=-3.04$ to -1.20$)$, negative symptoms $(\mathrm{WMD}=$ 2.96, $95 \% \mathrm{CI}=-4.09$ to -1.83 ) and general symptoms $(\mathrm{WMD}=-3.15,95 \% \mathrm{CI}=-4.21$ to -2.09$)$ (Table 2$)$.

\section{Psychosocial functioning}

A valid meta-analysis of psychosocial functioning could not be performed because of incomplete information for computing an effect size (Chabannes et al. 2008; PitschelWalz et al. 2006; Xiong et al. 1994) and the diversity of the scales used for measuring psychosocial functioning. Information on psychosocial functioning was obtained with the Global Assessment of Function Scale (GAF) (Merinder et al. 1999; Valencia et al. 2007; Vreeland et al. 2006; Xiong et al. 1994), the Global Assessment Scale (Hornung et al. 1996; Pitschel-Walz et al. 2006), the Social Functioning Schedule (Atkinson et al. 1996), the Social Adjustment Scale Self-Report (Chabannes et al. 2008), the Social Disability Screening Schedule (Xiang et al. 2006), and the Independent Living Skills Survey (Kopelowicz et al. 2003).

Visual inspection of the five studies revealed a statistically significant improvement in psychosocial functioning in the self-management education intervention groups compared with the control groups (Atkinson et al. 1996; Pitschel-Walz et al. 2006; Valencia et al. 2007; Xiang et al. 2006; Xiong et al. 1994). For instance, in Valencia et al.'s (2007) study, participants in a self-management education program demonstrated significant improvement in occupational functioning, social functioning, marital functioning, and money management compared to participants in the control group. In Pitschel-Walz et al.'s (2006) study, mean GAS scores of the self-management group and control group did not differ at baseline, but at the 12 months after the intervention, the mean score in the self-management group increased to 78 and in the control group to 68. In a Chinese study, Xiong et al. (1994) found that the duration of employment was longer in the selfmanagement group than in the control group at the 12and 18-month follow ups. In another Chinese study, Xiang et al. (2006) showed that participants in the selfmanagement group fared significantly better than their counterparts with respect to social functioning 6 months after intervention. In Atkinson et al.'s (1996) study, there was a significant increase in the number of social contacts, daily contacts, weekly contacts, and monthly contacts of those attending self-management groups. There was no significant increase in contacts in the control group.

In contrast, five other studies revealed no significant improvement in psychosocial functioning following selfmanagement education in participants receiving the intervention (Chabannes et al. 2008; Hornung et al. 1996; Kopelowicz et al. 2003; Merinder et al. 1999; Vreeland et al. 2006). Vreeland believed that the relatively short timeframe of their study may have limited the likelihood of observing significant changes in psychosocial functioning. In Merinder et al.'s (1999) study, no differences were found in psychosocial functioning between the selfmanagement group and the control group. The authors 
TABLE 3: Effect sizes and confidence intervals for relapse, re-hospitalization, and adherence to medication (comparison between $>10$ and $\leq 10$ intervention sessions)

\begin{tabular}{|c|c|c|c|c|c|}
\hline \multirow[b]{2}{*}{ Outcome category } & \multirow[b]{2}{*}{$k$} & \multicolumn{2}{|l|}{$n$} & \multirow[b]{2}{*}{ Total effect size $(95 \% \mathrm{CI}) \dagger$} & \multirow[b]{2}{*}{$P$} \\
\hline & & $\begin{array}{c}\text { Self-management } \\
\text { education intervention }\end{array}$ & $\begin{array}{c}\text { Treatment } \\
\text { as usual }\end{array}$ & & \\
\hline \multicolumn{6}{|l|}{ Intervention sessions $>10$} \\
\hline Relapse & 3 & 120 & 113 & $\mathrm{OR}=0.41(0.21-0.79)$ & 0.008 \\
\hline Re-hospitalization & 4 & 159 & 158 & $\mathrm{OR}=0.35(0.17-0.71)$ & 0.004 \\
\hline Adherence to medication & 2 & 82 & 84 & $\mathrm{OR}=1.79(0.76-4.19)$ & 0.080 \\
\hline \multicolumn{6}{|l|}{ Intervention sessions $\leq 10$} \\
\hline Relapse & 2 & 155 & 146 & $\mathrm{OR}=0.67(0.39-1.15)$ & 0.014 \\
\hline Re-hospitalization & 3 & 233 & 221 & $\mathrm{OR}=0.63(0.43-0.93)$ & 0.020 \\
\hline Adherence to medication & 2 & 147 & 122 & $\mathrm{OR}=3.08(1.69-5.61)$ & 0.000 \\
\hline
\end{tabular}

$\dagger$ Integration carried out in fixed effects model. CI, confidence interval; $k$, number of included studies; $n$, number of investigated participants; OR, odds ratio; $P$, level of significance for total effect size.

proposed that few published studies have shown an effect on psychosocial functioning and that the reason may be that these effects are indirect and delayed beyond the measurement of these studies.

\section{Efficacy of self-management education interventions (difference between interventions with $>10$ and $\leq 10$ sessions)}

Relapse

Among five studies reporting outcome data on relapse, three had more than 10 sessions and two studies had 10 sessions or less. When separately analyzed, it was found that those receiving more than 10 sessions were 59\% less likely to experience relapse than those receiving treatment as usual $(\mathrm{OR}=0.41,95 \% \mathrm{CI}=0.21-0.79$, $P=0.008)$. In contrast, those receiving 10 sessions or less were $33 \%$ less likely to experience relapse than those receiving treatment as usual $(\mathrm{OR}=0.67,95 \% \mathrm{CI}=0.39$ $1.15, P=0.014)$ (Table 3$)$.

\section{Re-hospitalization}

Among seven studies reporting outcome data on re-hospitalization, four had more than 10 sessions and three studies had 10 sessions or less. When separately analyzed, it was found that those receiving more than 10 sessions were $65 \%$ less likely to experience rehospitalization than those receiving treatment as usual $(\mathrm{OR}=0.35,95 \% \mathrm{CI}=0.17-0.71, P=0.004)$. In contrast, those receiving 10 sessions or less were $37 \%$ less likely to experience re-hospitalization than those receiving treatment as usual $(\mathrm{OR}=0.63,95 \% \mathrm{CI}=0.43-0.83, P=0.020)$

(Table 3).

\section{Adherence to medication}

Among four studies reported outcomes on adherence to medication, two had more than 10 sessions and two studies had 10 sessions or less. When separately analyzed, it was found that there was a 3.08-fold greater odds of adherence to medication for participants receiving 10 sessions or less than for those receiving treatment as usual $(\mathrm{OR}=3.08,95 \% \mathrm{CI}=1.69-5.61, P=0.000)$. However, there was no significant difference between participants receiving more than 10 sessions of self-management intervention than for those receiving treatment as usual $(\mathrm{OR}=1.79,95 \% \mathrm{CI}=0.76-4.19, P=0.08)($ Table 3$)$.

\section{DISCUSSION}

\section{Summary of results}

Difference between the current meta-analysis and other published reviews

There have been published reviews examining the effectiveness of psychoeducation for persons with schizophrenia such as those by Xia et al. (2011) and Lincoln et al. (2007). There are three major differences between the current meta-analysis and other studies. First, the current study involved studies that had to have included teaching self-management skills for persons with schizophrenia. In contrast, in other reviews, not every study included a self-management component. Some studies only provided participants with disease-specific information. Second, as mentioned earlier, there are many selfmanagement intervention programs at present. However, the content of these programs varies greatly. The current meta-analysis required that included studies be selfmanagement programs that included the four core areas of self-management: medication management, recognition of early warning signs of relapse, development of a relapse prevention plan, and coping skills for dealing with persistent symptoms based on Mueser and McGurk's recommendations (Mueser \& McGurk 2004). Third, the 
current meta-analysis investigated the impact of the intensity of intervention and provided preliminary support for interventions with more than 10 sessions as benefiting relapse and re-hospitalization. These findings provide recommendations to clinical nurses that patients should receive more intervention sessions in order to gain a benefit on relapse and re-hospitalization.

\section{Results of current meta-analysis}

This is the first meta-analysis of the efficacy of selfmanagement education intervention for persons with schizophrenia. This analysis provided limited evidence to support the work of Mueser and McGurk (2004), pointing to the effectiveness of self-management education as a psychosocial management strategy in its own right. Mueser and McGurk (2004) had advanced the notion that self-management education for persons suffering from schizophrenia must incorporate content pertaining to schizophrenia and its treatment, strategies for medication management; recognition of early warning signs of relapse and development of a relapse prevention plan; and coping skills for dealing with persistent symptoms. According to the meta-analysis, application of Meuser and McGurk's schema for self-management education led to three out of four measured outcomes: adherence to medication, fewer symptoms, and fewer relapses or re-hospitalization events. These findings can provide a foundation for further research on the efficacy of self-management education for persons suffering from schizophrenia.

Reduction of relapse and re-hospitalization in the selfmanagement education group may help explain the assertion that self-management education provides persons with schizophrenia a greater degree of autonomy and responsibility in dealing with their illness. It is possible that knowledge and techniques acquired through selfmanagement education enable and empower persons with schizophrenia not only to recognize environmental triggers and early warning signs of relapse, but also to implement relapse prevention plans earlier rather than later or not at all (Herz et al. 2000). It is also possible that better medication adherence helps to optimize relapse prevention strategies. The positive impact of selfmanagement education on adherence to medication thus may be a main effect of the intervention.

Nine studies measuring psychiatric symptoms with PANSS $(n=4)$ or BPRS $(n=5)$ demonstrated significant reductions in symptom severity among participants in the self-management education group compared to participants in the control group. The findings point to those participants being more able to use knowledge and skills gained through their education to manage some of their symptoms.

The effect of intervention intensity was examined by analyzing studies with more than 10 sessions and those with 10 or fewer sessions. Our study demonstrated that participants receiving more than 10 sessions were 59\% less likely to experience relapse and $65 \%$ less likely to experience re-hospitalization. In contrast, participants receiving 10 or fewer sessions were $33 \%$ less likely to experience relapse, $37 \%$ less likely to experience re-hospitalization. It seemed to show that participants receiving more than 10 sessions gained more benefit than those receiving 10 or less sessions in terms of relapse and re-hospitalization. It is possible that a relatively limited amount of self-management education intervention is insufficient to decrease relapse and re-hospitalization. The impact of intensity of self-management intervention on adherence to medication could not be concluded in this meta-analysis because there was no significant difference among participants receiving more than 10 intervention sessions. It is possible that with only two studies involved in this meta-analysis, it may not have been suitable for inclusion in the meta-analysis. The impact of intensity of self-management intervention on psychiatric symptoms could not be evaluated in this meta-analysis. In our study, there were four studies reporting mean data for psychiatric symptoms measured with the PANSS, however, those four studies all included more than 10 intervention sessions. Similarly, five studies reported mean data for psychiatric symptoms measured with the BPRS, however, all of these studies had fewer than 10 sessions. Therefore, it was not possible to examine differences between different intensity groups. In summary, our findings provide preliminary support for the benefits of self-management interventions with more than 10 sessions on relapse and re-hospitalization, and point to the need for more research on adherence to medication and psychiatric symptoms.

The current meta-analysis could not be performed for data on psychosocial functioning because the studies used different rating scales. There were not enough studies using a particular rating scale from which to assess functioning. Some studies, in addition, did not provide complete information for computing effect size.

\section{Heterogeneity}

The psychiatric symptoms measured with the PANSS in four studies was the only variable in the meta-analysis that demonstrated high statistical heterogeneity following self-management education. It is possible that time to follow-up measurement may have contributed to this 
finding because statistical heterogeneity was less evident when the same outcome for each study was examined separately. Noteworthy is the fact that Vreeland et al. (2006) did not obtain a fair assessment of participant change (or any success of self-management education) because they assessed participant symptoms only immediately after the intervention, whereas in the other three studies the researchers assessed participant symptoms at 6 or 12 months following intervention. Even so, it is also possible that in the Vreeland et al. (2006) study, participants were older and had greater baseline severity and variability of positive and negative symptoms than did participants in the other three studies and may have needed more time for the intervention to take. The participants in the education intervention group may have had more persistent symptoms, been, more resistant to treatment, or given more difficult content to master; or the interventionists may not have delivered the intervention adequately. Third, control group participants in the Vreeland et al. (2006) study received treatment in a university-based setting specializing in the treatment of persons with severe mental illness. The treatment as usual delivered may have been considerably more enriched than the treatment usually available to individuals with schizophrenia.

\section{Strengths and limitations}

There were several strengths of this review. First, the published work search was conducted thoroughly and systematically, including the search of several electronic databases, hand searching of major psychiatric journals, and the search of the reference lists of obtained publications. Second, the authors used a well-designed protocol to include or exclude studies on the basis of the design of an RCT, the content of a self-management education intervention, and diagnosis of participants. Third, the authors contacted authors of studies for missing data in an effort to reduce potential bias of the meta-analysis. Finally, the studies included in the meta-analysis originated from a variety of different settings, countries, and cultures, and participants of varying ages, making them potentially more able to be generalized. However, there are some limitations of this study:

\section{Internal validity of studies}

In this meta-analysis, there are no clear variations in subject characteristics between self-management intervention groups and control groups. For instance, males comprised $29.2-100 \%$ in the intervention groups and $38-100 \%$ in the control groups. The mean age of the participants ranged 29.7-39.5 years in the intervention groups and 30.1-40 years in the control groups. Attrition rates in the two groups were also comparable. In the intervention groups, attrition rates varied from $0-37 \%$, while in the control groups, attrition rates ranged $0-33 \%$. However, there is some variation in terms of the number and duration of intervention sessions and the time of follow-up assessments. Those might have influenced the outcomes of the studies included in the meta-analysis. However, because of a relatively small number of studies and a small overlap in outcome measures, subgroup analysis was not possible. For future research, it would be very interesting to perform subgroup analyses to investigate whether there were differences among different settings (community, outpatient, and inpatient), between different medications, between different numbers and durations of intervention sessions, between different sources of data (participant reports, clinician reports), and between studies that have greater or lesser numbers of sessions.

It should be noticed that the majority of the studies in this meta-analysis used subjective and indirect methods (clinician reports, self-reports, medical records) as measures of adherence to medication. Although they are the most commonly used methods, subjective assessments are likely to be inaccurate and tend to overestimate levels of adherence (Velligan et al. 2010). The use of direct or objective measures (e.g. pill count, blood or urine analysis, electronic monitoring, and electronic refill records) has the potential to provide much more reliable and accurate information (Velligan et al. 2010).

\section{Methodological quality of meta-analysis}

Although most studies in this meta-analysis scored above 3 based on the Jadad score, there are some issues of note. First, only five studies contained a description of the method of randomization. The method of generating the randomization sequence was not clear in the other eight studies. Second, three studies did not report whether they used blinding. Among 10 blinded studies, one study did not provide information about its method of blinding. In this meta-analysis, none of the studies were doubleblinded. According to Lorig (2003), self-management interventions are never double-blinded. It is impossible to blind study participants to determine whether they are receiving an intervention or which intervention they are receiving. Those giving the intervention likewise are not blinded. However, Lorig emphasized that outcome assessors as well as the investigators can be blinded. In most good self-management studies, outcome assessment is totally separated from the intervention (Lorig 2003). Third, two studies (Merinder et al. 1999; Shin \& Lukens 
2002) in this meta-analysis used small sample sizes limiting potential power of study findings. Fourth, complete data for computing effect size statistics were not available for some studies although this potential bias was reduced by personal contact with some of the authors for missing data. For example, three studies did not provide information about means or standard deviations of psychiatric symptoms, therefore, the effect size could not be calculated (Atkinson et al. 1996; Chabannes et al. 2008; Xiong et al. 1994). Fifth, meta-analyses could not be performed on certain outcomes (e.g. psychosocial functioning) because they were measured by using rating scales that were not comparable in meaning or scaling. This lack of available data not only hampers statistical analysis but also fails to provide a basis for advancing knowledge of selfmanagement education as an intervention in its own right. The possible 'file drawer' problem should also be considered when interpreting the outcomes of meta-analysis. Although the authors made every effort to ensure that relevant studies were included, it is difficult to know how many studies have been conducted but have never been reported. In other words, meta-analysis relies on published studies, which may create exaggerated outcomes. Finally, there may be a language bias as only studies published in English were reviewed.

\section{Implications for nursing practice}

The findings from the current meta-analysis do not refute the idea that delivery of a self-management education intervention can reduce relapse, re-hospitalization, and severity of psychiatric symptoms. The findings provide initial support for the feasibility of the intervention in terms of its potential for positive outcomes. For example, the results indicated that participants receiving selfmanagement education intervention were $46 \%$ less likely to experience relapse, $45 \%$ less likely to experience re-hospitalization, and had 2.57-fold greater odds of adherence to medication than those receiving treatment as usual. The findings also provided preliminary support for the benefits of self-management interventions with greater than 10 sessions on relapse and re-hospitalization. Although valid meta-analysis of psychosocial functioning could not be performed mainly because of the diversity of the scales used for measuring psychosocial functioning, visual inspection revealed that half of the included studies in this meta-analysis had statistically significant improvements in psychosocial functioning in the self-management education intervention groups compared with treatment as usual groups.

Our findings provide initial support for the efficacy of the self-management intervention. As many countries already routinely deliver self-management education interventions to persons with schizophrenia, clinical nurses should consider how to improve the interventions in order to help persons with schizophrenia gain more benefits from self-management intervention. Suggestions include:

1 Although there are many self-management programs for persons with schizophrenia, the content varied greatly. Nurses in different countries should focus on the core contents for those patients based on their cultural context.

2 The findings of this study provided preliminary support for the benefits of self-management intervention with greater than 10 sessions on relapse and rehospitalization. The findings imply that patients should receive adequate intervention sessions (at least 10 sessions) in future self-management intervention programs.

3 All studies in the currently meta-analysis were based on group face-to-face intervention. Future interventions should consider other types of format, such as Internetmediated programs and telephone counselling.

\section{Implications for research}

Researchers in different countries should consider developing standardized self-management education guidelines and training materials based on the context of their individual culture. The content of self-management programs is related to the cultural context. For instance, many self-management intervention programs in Western countries include coping with drug and alcohol addiction. However, addictions disorders are not common for most persons with schizophrenia in Asian countries. Therefore, few programs included coping with drug and alcohol use. The goal of developing standardized self-management education guidelines and training materials is to facilitate the faithful implementation of evidence-based practices and to improve patient outcomes in routine mental health service settings.

Second, researchers especially need to improve on the design (internal validity) of efficacy studies of selfmanagement education for persons with schizophrenia by controlling for sources of bias. For example, further research on the efficacy of self-management education interventions should be conducted in well-designed RCT with a long follow-up period so that definite conclusions can be made. Researchers should make every effort to minimize bias by using blind or independent raters. Larger samples are also needed to ensure adequate power to detect clinically important effects. Third, the use of a 
therapy manual or protocol should be considered, so that adherence to the model can increase internal validity and consistency of delivery of the intervention. Fourth, future research should not only include compliance with medication, relapse and readmission, symptoms, and psychosocial functioning, but also quality of life, treatment satisfaction, adverse events (suicide attempts or violence), and other important measures of self-management programs, such as self-efficacy, empowerment, insight into illness, and sense of coherence. Health economic outcomes should also be measured, as the efficiency of selfmanagement is crucial in making it an attractive option for managers and policy makers. Finally, researchers could try to examine outcomes of peer-led self-management education interventions in persons with schizophrenia in future studies.

\section{ACKNOWLEDGEMENT}

This research was funded by the China Medical Board (grant no. 04-797).

\section{REFERENCES}

Angell, B. \& Test, M. A. (2002). The relationship of clinical factors and environmental opportunities to social functioning in young adults with schizophrenia. Schizophrenia Bulletin, 28 (2), 259-271.

Anzai, N., Yoneda, S., Kumagai, N., Nakamura, Y., Ikebuchi, E. \& Liberman, R. P. (2002). Training persons with schizophrenia in illness self-management: A randomized controlled trial in Japan. Psychiatric Services, 53 (5), 545-547.

Atkinson, J. M., Coia, D. A., Gilmour, W. H. \& Harper, J. P. (1996). The impact of education groups for people with schizophrenia on social functioning and quality of life. British Journal of Psychiatry, 168 (2), 199-204.

Bodenheimer, T., Lorig, K., Holman, H. \& Grumbach, K. (2002). Patient self-management of chronic disease in primary care. Journal of the American Medicine Association, 288 (19), 2469-2475.

Bourbeau, J., Julien, M., Maltais, F. et al. (2003). Reduction of hospital utilization in patients with chronic obstructive pulmonary disease: A disease-specific self-management intervention. Archives of Internal Medicine, 163 (5), 585591.

Chabannes, J. P., Bazin, N., Leguay, D. et al. (2008). Two-year study of relapse prevention by a new education program in schizophrenic patients treated with the same antipsychotic drug. European Psychiatry, 23 (1), 8-13.

Chan, S. H., Lee, S. W. \& Chan, I. W. (2007). TRIP: A psychoeducational programme in Hong Kong for people with schizophrenia. Occupational Therapy International, 14 (2), 86-98.
Chan, S. W., Yip, B., Tso, S., Cheng, B. S. \& Tam, W. (2009). Evaluation of a psychoeducation program for Chinese clients with schizophrenia and their family caregivers. Patient Education and Counseling, 75 (1), 67-76.

Herz, M. I., Lamberti, J. S., Mintz, J. et al. (2000). A program for relapse prevention in schizophrenia: A controlled study. Archives of General Psychiatry, 57 (3), 277-283.

Higgins, J. P., Thompson, S. G., Deeks, J. J. \& Altman, D. G. (2003). Measuring inconsistency in meta-analyses. British Medical Journal, 327 (7414), 557-560.

Hornung, W. P., Kieserg, A., Feldmann, R. \& Buchkremer, G. (1996). Psychoeducational training for schizophrenic patients: Background, procedure and empirical findings. Patient Education and Counseling, 29 (3), 257-268.

Kane, J. M. (1996). Treatment-resistant schizophrenic patients. Journal of Clinical Psychiatry, 57 (Suppl. 9), 35-40.

Kopelowicz, A., Zarate, R., Gonzalez, S. V., Mintz, J. \& Liberman, R. P. (2003). Disease management in Latinos with schizophrenia: A family-assisted, skills training approach. Schizophrenia Bulletin, 29 (2), 211-227.

Lincoln, T. M., Wilhelm, K. \& Nestoriuc, Y. (2007). Effectiveness of psychoeducation for relapse, symptoms, knowledge, adherence and functioning in psychotic disorders: A meta-analysis. Schizophrenia Research, 96 (1-3), 232-245.

Lindenmayer, J. P. (2000). Treatment refractory schizophrenia. The Psychiatric Quarterly, 71 (4), 373-384.

Lorig, K. (2003). Self-management education: More than a nice extra. Medical Care, 41 (6), 699-701.

McGurk, S. R. \& Mueser, K. T. (2004). Cognitive functioning, symptoms, and work in supported employment: A review and heuristic model. Schizophrenia Research, 70 (2), 147173.

Meder, J., Morawiec, M. \& Sawicka, M. (1998). Evaluation of the medication management module in Poland. International Review of Psychiatry, 10 (1), 62-66.

Merinder, L. B., Viuff, A. G., Laugesen, H. D., Clemmensen, K., Misfelt, S. \& Espensen, B. (1999). Patient and relative education in community psychiatry: A randomized controlled trial regarding its effectiveness. Social Psychiatry and Psychiatric Epidemiology, 34 (6), 287-294.

Mueser, K. T. \& McGurk, S. R. (2004). Schizophrenia. Lancet, 363 (9426), 2063-2072.

Newman, S., Steed, L. \& Mulligan, K. (2004). Self-management interventions for chronic illness. Lancet, 364 (9444), 15231537.

Norman, R. M., Malla, A. K., Cortese, L. et al. (1999). Symptoms and cognition as predictors of community functioning: A prospective analysis. American Journal of Psychiatry, 156 (3), 400-405.

Pitschel-Walz, G., Bauml, J., Bender, W., Engel, R. R., Wagner, M. \& Kissling, W. (2006). Psychoeducation and compliance in the treatment of schizophrenia: Results of the Munich Psychosis Information Project Study. Journal of Clinical Psychiatry, 67 (3), 443-452. 
Racenstein, J. M., Harrow, M., Reed, R., Martin, E., Herbener, E. \& Penn, D. L. (2002). The relationship between positive symptoms and instrumental work functioning in schizophrenia: A 10 year follow-up study. Schizophrenia Research, 56 (1), 95-103.

Shin, S. \& Lukens, E. P. (2002). Effects of psychoeducation for Korean Americans with chronic mental illness. Psychiatric Services, 53 (9), 1125-1131.

Valencia, M., Rascon, M. L., Juarez, F. \& Murow, E. (2007). A psychosocial skills training approach in Mexican out-patients with schizophrenia. Psychological Medicine, 37 (10), 13931402.

Velligan, D. I., Weiden, P. J., Sajatovic, M. et al. (2010). Strategies for addressing adherence problems in patients with serious and persistent mental illness: Recommendations from the expert consensus guidelines. Journal of Psychiatric Practice, 16 (5), 306-324.

Vreeland, B., Minsky, S., Yanos, P. T. et al. (2006). Efficacy of the team solutions program for educating patients about illness management and treatment. Psychiatric Services, 57 (6), 822-828.
World Health Organization (2001). The WHO World Health Report: New Understanding, New Hope. Geneva: World Health Organization. Schizophrenia. [Cited July 2009]. Available from: URL: http://www.who.int/mental_health/ management/schizophrenia/en/

Wu, E. Q., Birnbaum, H. G., Shi, L. et al. (2005). The economic burden of schizophrenia in the United States in 2002. Journal of Clinical Psychiatry, 66 (9), 1122-1129.

Xia, J., Merinder, L. B. \& Belgamwar, M. R. (2011). Psychoeducation for schizophrenia. Cochrane Database of Systematic Reviews, (6), CD002831. DOI: 10.1002/14651858. CD002831.pub2.

Xiang, Y., Weng, Y., Li, W. et al. (2006). Training patients with schizophrenia with the community re-entry module: A controlled study. Social Psychiatry and Psychiatric Epidemiology, 41 (6), 464-469.

Xiong, W., Phillips, M. R., Hu, X. \& Wang, R. (1994). Familybased intervention for schizophrenic patients in China: A randomised controlled trial. British Journal of Psychiatry, 165 (2), 239-247. 